\title{
Применение методов хромато-масс-спектрометрии в целях проведения комплексной посмертной химико-токсикологической экспертизы
}

\author{
Темердашев А.З., Гашимова Э.М. \\ Кубанский государственный университет, Краснодар \\ Поступила в редакцию 23.08.2019 г.
}

DOI: 10.17308/sorpchrom.2019.19/1168

В данной статье рассмотрен случай внезапной смерти, вероятной причиной которой стало сочетание употребления алкоголя, эфедрина и анаболических стероидов в сочетании с систематическими тяжелыми тренировками. В результате посмертной экспертизы, проведенной с использованием ультравысокоэффективной жидкостной хроматографии в сочетании $\mathrm{c}$ тандемным массспектрометрическим детектированием, газовой хромато-масс-спектрометрии с источником электронной ионизации и газовой хроматографии с пламенно-ионизационным детектором и дозированием равновесной паровой фазы.

Ключевые слова: эфедрин, этанол, стероиды, тестостерон, УВЭЖХ-МС/MС, ГХ-МС.

\section{Application of chromatography and mass-spectrometry for the complex toxicological expertices}

\author{
Temerdashev A.Z., Gashimova E.M.
}

\section{Kuban State University, Krasnodar}

In recent years, the promotion of sports and healthy lifestyles has been conducted as part of governmental programs all over the world. The number of people involved in sports is steadily growing and as a consequence a shadow market of performance-enhancing drugs and supplements also increases. Such drugs often contain compounds banned by the World Anti-Doping Agency (WADA).

A wide range of compounds is included in the Prohibited list: stimulants, anabolic agents, different non-approved substances, etc., however, they are still available on the free market and can be found even in sports nutrition. The temptation to experiment with these supplements is huge for lots of athletes. Moreover, manufacturers claim the necessity of food supplements in order to perform better and to be competitive. As a result, even anabolic agents can be found in sports nutrition. For the class of "fatburners" the presence of prohibited ephedrine, synephrine and methylsynephrine is common. In some cases, supplements contained banned substances not indicated on the label.

An unexpected death case is presented in this article. It might have happened because of an unacceptable combination of alcohol, ephedrine, anabolic agents and heavy training. The results of post-mortem expertise using ultra-high performance liquid chromatography-tandem mass-spectrometry (UHPLC-MS/MS), gas chromatography-mass spectrometry (GC-MS) and headspace gas chromatography combined with flame ionization detection (HS-GC-FID) are presented. The presence of exogenous testosterone in urine was suspected due to abnormally high testosterone/epitestosterone ratio (9:1) along with the total testosterone concentration of $130 \mathrm{ng} / \mathrm{mL}$. Ephedrine was found as a component of a fatburner and its concentration in urine was $12 \mu \mathrm{g} / \mathrm{mL}$. The presence of ethanol in blood was confirmed by HS-GC-FID and its concentration was $0.3 \mathrm{mg} / \mathrm{L}$. LODs for ephedrine and testosterone evaluated by UHPLC-MS/MS determination in urine were $0.005 \mu \mathrm{g} / \mathrm{mL}$ and $0.05 \mathrm{ng} / \mathrm{mL}$, respectively. LOQ for HS-GC-FID determination of ethanol in blood was 0.1 $\mathrm{mg} / \mathrm{L}$.

Keywords: ephedrine, ethanol, steroids, testosterone, UHPLC-MS/MS, GC-MS 


\section{Введение}

В последние годы количество программ поддержки здорового образа жизни во всем мире существенно увеличилось, что привело не только к росту числа людей, занимающихся спортом, но и распространению различных препаратов и средств, позволяющих повысить эффективность тренировок, с целью скорейшего достижения результата. При этом целый ряд подобных средств может содержать вещества, запрещенные Всемирным Антидопинговым Агенством (ВАДА) [1,2].

Список подобных соединений представлен в перечне запрещенных препаратов и включает в себя стимуляторы, андрогенные анаболические стероиды, модуляторы метаболизма, различные вещества, не прошедшие сертификацию и клинические испытания. В ряде случаев продукты спортивного питания могут содержать подобные вещества, при этом они могут быть не указаны в составе, что делает их контроль особенно актуальным, поскольку согласно кодексу ВАДА именно спортсмен несет ответственность за обнаружение в его организме запрещенных субстанций даже в случае отсутствия умысла в их употреблении. Так, например, для класса жиросжигателей характерно присутствие эфедрина, синефрина и метилсинефрина [3-7].

В ряде случаев, желая быстро достигнуть нужного результата, спортсмены экспериментируют с различными сочетаниями запрещенных препаратов. К сожалению, зачастую подобные эксперименты не имеют под собой разработанной спортивными врачами и другими специалистами схемы употребления и базируются исключительно на субъективных ощущениях человека, употребляющего эти препараты. Подобное бесконтрольное употребление может приводить к необратимым повреждениям организма.

На сегодняшний день, прохождение спортсменами процедуры электрокардиографии (ЭКГ), стало обязательным. Это позволяет диагностировать проблемы с сердцем на ранних стадиях, но, к сожалению, даже сейчас количество внезапных смертей, обусловленных проблемами с сердцем, достаточно велико [8,9]. В случае возникновения подобных ситуаций, особенно важно понять, что стало причиной смерти, что требует проведения полного спектра токсикологических исследований.

Целью данной работы является разработка и применение ряда методик качественного и количественного анализа, позволяющих проводить комплексные химико-токсикологические исследования с использованием ультравысокоэффективной жидкостной хроматографии с тандемным масс-спектрометрическим детектированием (УВЭЖХ-МС/МС) для определения тестостерона, установления соотношения тестостерона/эпитестостерон и определения эфедрина в моче, газовой хромато-массспектрометрии для анализа спортивного питания (ГХ-МС) и газовой хроматографии с пламенно-ионизационным детектированием и дозированием равновесной паровой фазы (ГХ-ПИД-ДРП) для определения содержания этанола в крови [10-13], с целью обеспечения высокой надежности и чувствительности при проведении исследований.

Рассматриваемый случай. 28-летний мужчина, занимающийся тяжелой атлетикой, был обнаружен мертвым в квартире. В ходе проведения осмотра помещения, сотрудниками полиции были обнаружены различные виды спортивного питания и ампулы с маслянистой жидкостью. Согласно заключению из морга, причиной внезапной смерти стала остановка сердца. Из протокола опроса родственников известно, что мужчина был абсолютно здоров, но постоянно употреблял различное спортивное питание. Образцы замороженной мочи, трупной крови и спортивного питания были отправлены в лабораторию для проведения анализа. 


\section{Эксперимент}

Реактивы и реагенты. Эфедрин, фенибут, метилтестостерон и тестостерон (>99\%) были приобретены у Sigma (США). Стандартные образцы растворов этанола получены от компании «Мониторинг» (Россия). В качестве растворителей использовалась вода (18.2 M $\Omega$ ), очищенная с использованием системы Milli-Q, Millipore (Франция), метанол и ацетонитрил, квалификации «для ВЭЖХ» приобретены у Sigma-Aldrich (США). Муравьиная кислота (>99\%, чистоты для синтеза) и диэтиловый эфир были предоставлены Acros Organics (США).

Приборы и оборудование.

УВЭЖХ-МС/MC. Ультравысокоэффективный жидкостный хроматограф Thermo Ultimate-3000 (Германия), соединенный с тандемным массспектрометрическим детектором Thermo TSQ Quantum Access Max (США), оснащенный источником нагреваемой электрораспылительной ионизации HESI-II (HЭРИ), использовался в ходе проведения исследования. Температура источника ионизации $-420^{\circ} \mathrm{C}$, трансферного капилляра $-320^{\circ} \mathrm{C}$, поток оболочного газа (азота) -50 у.е., вспомогательного газа (азота) - 15 у.е., напряжение на источнике ионизации - 4 кВ, давление газа-мишени (аргон) в ячейке соударений - 1.5 мТорра.

Разделение осуществляли с помощью аналитической колонки Phenomenex Kinetex $\mathrm{C}_{18}(75 \times 2.1$ мм, 1.7 мкм) в режиме обращенно-фазовой жидкостной хроматографии. В качестве подвижной фазы использовали метанол (А) и $0.1 \%$ водный раствор муравьиной кислоты (В). Скорость потока подвижной фазы $-0.4 \mathrm{~cm}^{3} /$ мин. Элюирование градиентное, двухступенчатое. Первая ступень: плато 1 мин 95\% В, $5 \%$ А, с последующим линейным увеличением содержания метанола в составе подвижной фазы до $90 \%$ за 2.5 мин и плато в течение 4 мин. Возврат к элюенту первой ступени осуществлялся за 0.5 мин с последующей регенерацией колонки (1 мин).

Детектирование осуществляли в режиме множественного мониторинга реакций (ММР). Переходы и энергии соударений приведены в табл. 1.

Таблица 1. MRM-переходы аналитов

\begin{tabular}{|c|c|c|c|c|c|}
\hline Вещество & $\mathrm{R}_{\mathrm{t}}$, мин & $\begin{array}{c}\text { Напряжение на } \\
\text { экстрагирующей } \\
\text { линзе, V }\end{array}$ & $\begin{array}{c}\text { Ион- } \\
\text { прекурсор, } \\
\mathrm{m} / \mathrm{z}\end{array}$ & $\begin{array}{l}\text { Энергия со- } \\
\text { ударения, eV }\end{array}$ & $\begin{array}{c}\text { Ион- } \\
\text { продукт, } \\
\mathrm{m} / \mathrm{z}\end{array}$ \\
\hline \multirow{3}{*}{ Эфедрин } & \multirow{3}{*}{1.84} & \multirow{3}{*}{ (n) } & \multirow{3}{*}{166.1} & 27 & $115.1(\mathrm{q})$ \\
\hline & & & & 33 & $91.2(\mathrm{~d})$ \\
\hline & & & & 17 & $133.1(\mathrm{~d})$ \\
\hline \multirow{3}{*}{ Фенибут } & \multirow{3}{*}{0.60} & \multirow{3}{*}{41} & \multirow{3}{*}{180.1} & 35 & $91(\mathrm{~d})$ \\
\hline & & & & 17 & $117(\mathrm{q})$ \\
\hline & & & & 12 & 145 (d) \\
\hline \multirow{3}{*}{ Тестостерон } & \multirow{3}{*}{6.63} & \multirow{3}{*}{78} & \multirow{3}{*}{289.2} & 23 & $97.2(\mathrm{q})$ \\
\hline & & & & 25 & $109.1(\mathrm{~d})$ \\
\hline & & & & 36 & $79.2(\mathrm{~d})$ \\
\hline \multirow{3}{*}{ Эпитестостерон } & \multirow{3}{*}{6.70} & \multirow{3}{*}{78} & \multirow{3}{*}{289.2} & 23 & $97.2(q)$ \\
\hline & & & & 25 & $109.1(\mathrm{~d})$ \\
\hline & & & & 36 & $79.2(\mathrm{~d})$ \\
\hline \multirow{3}{*}{$\begin{array}{l}\text { Метилтестостерон } \\
\text { (ISTD) }\end{array}$} & \multirow{3}{*}{6.95} & \multirow{3}{*}{83} & \multirow{3}{*}{303.2} & 27 & $109.1(q)$ \\
\hline & & & & 27 & $97.1(\mathrm{~d})$ \\
\hline & & & & 42 & $79.1(\mathrm{~d})$ \\
\hline
\end{tabular}

$\mathrm{q}$ - ион для количественного анализа, $\mathrm{d}$ - диагностический ион

Управление прибором, сбор данных и их обработка проводилась с использованием программного обеспечения Thermo XCalibur 2.2. 
ГХ-МС. Для проведения исследований использовалась система, состоящая из газового хрмоатографа Thermo Trace-1310, оснащенного капиллярной колонкой Phenomenex Zebron ZB-5MS (30 м×0.25 мм×0.25 мкм). Скорость потока газа-носителя (гелий 6.0) составляла $1 \mathrm{~cm}^{3} /$ мин, температура инжектора $-250^{\circ} \mathrm{C}$, объем вводимой пробы - 1 мкл, режим ввода пробы - с делением потока, коэффициент деления потока - 20:1. Начальная температура термостата колонок составляла $60^{\circ} \mathrm{C}$ в течении 3 мин, с последующим нагревом до $150^{\circ} \mathrm{C}$ со скоростью $10^{\circ} \mathrm{C} /$ мин и нагревом до $270^{\circ} \mathrm{C}$ со скоростью $15^{\circ} \mathrm{C} /$ мин и плато 10 мин.

Газовый хроматограф был соединен с масс-спектрометром Thermo TSQ Quantum XLS, оснащенным источником электронной ионизации. Температура источника ионизации $-220^{\circ} \mathrm{C}$, трансферной линии $-280^{\circ} \mathrm{C}$, энергия электронов -70 эВ, диапазон сканирования масс - от 45 до 700 Да, время сканирования - 300 мс.

ГХ-ПИД-ДРП. Для разделения использовался газовый хроматограф Shimadzu GC-2010, оснащенный колонкой Agilent FFAP (50 м×0.32 мм×0.5 мкм). Скорость потока газа-носителя (азота) составляла $1.2 \mathrm{~cm}^{3} /$ мин Температура инжектора и детектора составляли 250 и $280^{\circ} \mathrm{C}$ соответственно. Начальная температура термостата составляла $40^{\circ} \mathrm{C}$, с последующим нагревом со скоростью $12^{\circ} \mathrm{C} /$ мин до $220^{\circ} \mathrm{C}$ и последующим плато в течении 5 мин. Газовый хроматограф был соединен с автоматическим дозатором AOC-5000 Shimadzu (CTC Analytics), оснащенным модулями нагрева и дозирования равновесной паровой фазы. Подготовка пробы осуществлялась в следующих условиях: температура термостатирования образца $-50^{\circ} \mathrm{C}$, время термостатирования - 20 мин, объем вводимой пробы $-0.1 \mathrm{~cm}^{3}$.

\section{Обсуждение результатов}

\section{Подготовка проб к анализу}

Определение эфедрина методом «разбавил и вколол» (УВЭЖХ-МС/МС). Аликвоту мочи, объемом 100 мкл, разбавляли 400 мкл смеси, состоящей из ацетонитрила и $0.1 \%$ водного раствора муравьиной кислоты (80:20, v:v) с внесением внутреннего стандарта (ISTD) - фенибута и центрифугировали со скоростью 11000 об/мин в течении 10 мин для осаждения белков. Супернатант отбирали и переносили в стеклянные виалы.

Определение стероидов в моче с использованием ферментативного гидролиза и жидкость-жидкостной экстракции (ЖЖЭ) (УВЭЖХ-МС/МС). В аликвоту мочи, объемом $2 \mathrm{~cm}^{3}$, добавляли $1 \mathrm{~cm}^{3}$ фосфатного буфера (рН 6.3), содержащего 30 мкл $\beta$-глюкуронидазы E.coli и метилтестостерон (ISTD2), с концентрацией 50 нг/см ${ }^{3}$ для проведения ферментативного гидролиза. Деконъюгация проводилась 1 час при температуре $57^{\circ} \mathrm{C}$, после чего к полученной пробе добавляли $1 \mathrm{~cm}^{3}$ карбонатного буфера (pH 10), 2 г безводного сульфата натрия и $3 \mathrm{~cm}^{3}$ диэтилового эфира, перемешивали 20 мин и центрифугировали 5 мин при 4000 об/мин. Водный слой вымораживали в криостате при $-25^{\circ} \mathrm{C}$, отделяли эфирный слой и упаривали его при $70^{\circ} \mathrm{C}$. Сухой остаток перерастворяли в 200 мкл смеси $0.1 \%$ водного раствора муравьиной кислоты и метанола (50:50, v:v).

Определение этилового спирта методом ГХ-ПИД с дозированием равновесной паровой фазы. 100 мкл образца крови разбавляли $1 \mathrm{~cm}^{3}$ смеси, содержащего внутренний стандарт $(0.01 \%$ раствор пропанола в воде) и переносили в виалу, объемом $20 \mathrm{~cm}^{3}$.

Установление метрологических характеристик методики. Оценка метрологических характеристик методик (табл. 2) проводилась с использованием 20 случайно 
выбранных холостых образцов мочи, полученных от добровольцев различных возрастных категорий и гендерной принадлежности.

Таблица 2. Некоторые метрологические характеристики методики

\begin{tabular}{|c|c|c|c|c|c|c|c|}
\hline \multirow[b]{2}{*}{ Вещество } & \multirow{2}{*}{$\begin{array}{c}\text { Предел } \\
\text { обна- } \\
\text { ру- } \\
\text { жения } \\
\left(\text { нг/см }{ }^{3}\right) \\
\end{array}$} & \multirow{2}{*}{$\begin{array}{c}\text { Линейный } \\
\text { диапазон } \\
(\text { нг/см³ })\end{array}$} & \multicolumn{2}{|c|}{$\begin{array}{c}\text { Воспроизводимость в } \\
\text { один день }\end{array}$} & \multicolumn{2}{|c|}{$\begin{array}{c}\text { Воспроизводимость в } \\
\text { разные дни }\end{array}$} & \multirow{2}{*}{$\begin{array}{c}\text { Матрич- } \\
\text { ные эф- } \\
\text { фекты, } \\
\%\end{array}$} \\
\hline & & & $\begin{array}{c}\text { Концентрация, } \\
\text { нг/мл }\end{array}$ & $\begin{array}{c}\text { СКО, } \\
\%\end{array}$ & $\begin{array}{c}\text { Концентрация, } \\
\mathrm{Hг} / \mathrm{cm}^{3}\end{array}$ & СКО, & \\
\hline \multirow{3}{*}{ Тестостерон } & \multirow{3}{*}{0.05} & \multirow{3}{*}{$\begin{array}{l}0.05-200 \\
(\mathrm{r}=0.997)\end{array}$} & 5 & 12.4 & 5 & 15.2 & \multirow{3}{*}{10} \\
\hline & & & 50 & 9.2 & 50 & 11.5 & \\
\hline & & & 200 & 6.3 & 200 & 7.9 & \\
\hline \multirow{3}{*}{$\begin{array}{c}\text { Эфедрин } \\
\text { («Разбавил } \\
\text { и вколол») }\end{array}$} & \multirow{3}{*}{1} & \multirow{3}{*}{$\begin{array}{c}1-2000 \\
(\mathrm{r}=0.991)\end{array}$} & 10 & 18.3 & 5 & 19.1 & \multirow{3}{*}{15} \\
\hline & & & 250 & 12.2 & 250 & 14.2 & \\
\hline & & & 1000 & 8.4 & 1000 & 9.3 & \\
\hline \multirow{3}{*}{$\begin{array}{l}\text { Эфедрин } \\
\text { (LLE) }\end{array}$} & \multirow{3}{*}{1} & \multirow{3}{*}{$\begin{array}{l}1-2000 \\
(\mathrm{r}=0.995)\end{array}$} & 10 & 14.7 & 5 & 16.4 & \multirow{3}{*}{10} \\
\hline & & & 250 & 10.6 & 250 & 12.7 & \\
\hline & & & 1000 & 9.8 & 1000 & 10.8 & \\
\hline \multirow{2}{*}{ Этанол } & \multirow[b]{2}{*}{-} & \multirow{2}{*}{$\begin{array}{l}100-2000 \\
(\mathrm{r}=0.998)\end{array}$} & 0.2 & 4.2 & 0.2 & 6.3 & \multirow[b]{2}{*}{-} \\
\hline & & & 0.8 & 2.9 & 0.8 & 4.1 & \\
\hline
\end{tabular}

Анализ продуктов спортивного питания. Полученные в ходе осмотра помещения образцы спортивного питания, были переданы в лабораторию для проведения исследования. Среди них присутствовали жиросжигатель «Hellfire» и предтренировочный комплекс «Fierce Domination». Навеска каждого образца, массой 0.1 г, экстрагировалась $20 \mathrm{~cm}^{3}$ водно-аммиачной смеси ( $\mathrm{pH}$ 9) в течении 30 мин при температуpe $45^{\circ} \mathrm{C}$ с последующей экстракцией $3 \mathrm{~cm}^{3}$ диэтилового эфира и центрифугированием в течение 5 мин при 3000 об/мин. Органический слой отбирали и упаривали в токе азота, после чего сухой остаток перерастворяли в 200 мкл смеси метанола и анализировали с использованием ГХ-МС в условиях, описанных выше, в режиме сканирования полного ионного тока. В результате было установлено, что предтренировчный комплекс не содержит запрещенных веществ, в то время как в образце жиросжигателя, предположительно, был обнаружен эфедрин, что потребовало проведения подтверждающего исследования с использованием УВЭЖХ-МС/МС.

Для этого навеску образца жиросжигателя, массой 0.1 г, экстрагировали смесью этанола с подкисленной 0.05 М соляной кислотой водой $(30: 70, \mathrm{v}: \mathrm{v})$ при $40{ }^{\circ} \mathrm{C}$ в течение 40 мин, после чего центрифугировали 5 мин при 3000 об/мин, переносили $1 \mathrm{~cm}^{3}$ экстракта в стеклянную виалу и анализировали в условиях, описанных выше.

Стеклянная ампула, содержащая маслянистую жидкость, предположительно, раствор анаболического стероида или их смесь в инъекционной форме, также подвергалась анализу с использованием метода ГХ-МС. Для этого аликвоту образца, объемом 10 мкл, растворяли в $1 \mathrm{~cm}^{3}$ диэтилового эфира и анализировали с использованием метода ГХ-МС в описанных выше условиях. Идентификация соединения проводилась путем сопоставления индексов удерживания аналита и его масс-спектра с библиотечными значениями. В результате проведенного исследования, было установлено, что вероятно, в ампуле содержится тестостерон деканоат. Поскольку данное исследование проводилось без использования стандартного образца аналита, идентификация носит исключительно вероятностный характер, позволяя установить исключительно вещество-кандидат.

Как правило, курсовое употребление анаболических стероидов исключает совмещение его с жиросжигателями, однако наличие обоих продуктов в изъятых мате- 
риалах требуют проведения исследования с целью определения в биологических жидкостях всех веществ, которые могли быть, потенциально, употреблены.

Принимая во внимание то, что в организме тестостерон деканоат гидролизуется до тестостерона и выводится из организма в конъюгированной форме, в целях его определения целесообразно проведение предварительной стадии деконъюгации с использованием ферментативного гидролиза, что позволяет избежать деградации аналитов, поскольку, в отличии от минерального гидролиза, он проводится в существенно более мягких условиях, описанных ранее. Помимо этого, употребление подобных препаратов приводит к нарушению эндогенного соотношения T/E, что также может свидетельствовать об употреблении экзогенных стероидов, поскольку согласно результатам популяционных исследований, эндогенные соотношение Т/Е, как правило, не превышает 4. Исключение могут составлять представители народов Кавказа, у которых данное соотношение может доходить до 6. Превышение этого значения в 95\% случаев свидетельствует о приеме экзогенного тестостерона [14].

Анализ образца мочи. К сожалению, в случае проведения посмертных экспертиз, объем мочи, которая может быть отобрана, крайне мал, что существенно ограничивает возможности применения ряда методов в целях проведения исследований. В данном случае, предоставленного образца было недостаточно для проведения подтверждающего исследования с использованием ГХ-МС, однако позволил провести полный цикл исследований с использованием ВЭЖХ-МС/МС (табл. 2).

Основным преимуществом процедуры «разбавил и вколол» является возможность быстрого скрининга большинства стимуляторов, которые могут быть обнаружены в нативной форме в моче. К такому типу соединений относится и эфедрин. Кроме того, применение этого способа позволяет избежать каких-либо потерь аналитов на этапе пробоподготовки. В результате проведенных исследований было ус-

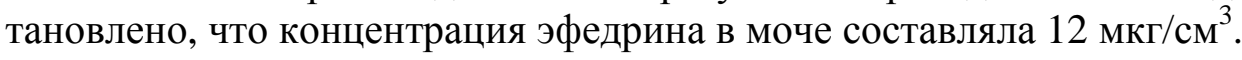

В то время как для определения тестостерона проведение предварительного гидролиза с последующей экстракцией было необходимо.

Принимая во внимание одинаковую эффективность ионизации тестостерона и эпитестостерона, для проведения количественного анализа и установления соотношения между ними достаточно было одного стандартного образца тестостерона. В результате проведенного исследования было установлено, что соотношение T/E coставляет 9:1, а концентрация тестостерона в исследуемом образце равна $130 \mathrm{Hг} / \mathrm{cm}^{3}$, что позволяет также позволяет предположить факт употребления андрогенных анаболических стероидов. К сожалению, ввиду отсутствия необходимого объема пробы мочи, провести подтверждение с использованием изотопной масс-спектрометрии, в соответствии с требованиями ВАДА, не представлялось возможным.

Анализ образца крови. Для построения калибровочных кривых использовалась цельная кровь, полученная от добровольцев, не употреблявших алкоголь более 2-х недель. Холостой образец готовили с использованием дистиллированной воды.

Головной раствор смеси, содержащей метанол, этанол и ацетон, с концентрацией 10 мг/дм ${ }^{3}$ вносили в цельную кровь добровольцев, после чего разбавляли водой, содержащей внутренний стандарт (н-пропанол) с целью приготовления серии калибровочных растворов с концентрациями аналитов $2,1,0.5,0.25$ и 0.1 мг/дм ${ }^{3}$. Количественный анализ проводился путем измерения соотношения факторов отклика этанола к н-пропанолу. В результате проведенного исследования было установлено, что концентрация этанола в крови составляла 0.3 мг/дм³ ${ }^{3}$ Принимая во внимание возраст и состояние здоровья умершего, данная концентрация достаточно мала и не могла сама по себе явиться причиной смерти. Однако в сочетании с систематическим употреблением жиросжигателей, содержащих эфедрин, анаболических стерои- 
дов и тяжелых физических нагрузок употребление алкоголя могло привести к летальному исходу.

\section{Заключение}

Показана возможность проведения комплексных экспертиз с использованием различных хроматографических и хромато-масс-спектрометрических методов анализа, отвечающих требованиям как высокой надежности, получаемых результатов, так и чувствительности и селективности, позволяющих проводить исследования в сложных матрицах.

Данная работа выполнена в рамках проекта № 4.2612.2017/ПЧ Минобрнауки РФ и при финансовой поддержке РФФИ, проект № 19-43-230004 p_a, с использованием научного оборудования ЦКП “Эколого-аналитический центр” Кубанского государственного университета, уникальный идентификатор иентра RFMEFI59317X0008.

\section{Список литературы}

1. World Anti-Doping Agency. The World Anti-Doping Code. The 2019 Prohibited List. International Standard. Режим доступа: https://www.wada-

ama.org/sites/default/files/wada_2019_english_ prohibited_list.pdf (дата обращения: 22.08.2019).

2. Thevis M., Kuuranne T., Geyer H. // Drug Test. Anal. 2018. Vol. 10. pp. 9-27.

3. De Cock K.J.S., Delbeke F.T., Van Eenoo P., Desmet N. et al. // J. Pharm. Biomed. Anal. 2001. Vol. 25. pp. 843-852.

4. de Hon O., Coumans B. // British J. Sports Med. 2007. Vol. 41. pp. 800-805.

5. Furjan Mandic G., Peric M., Krzelj L., Stankovic S. et al. // J. Sports Sci. Med. 2013. Vol. 12. pp. 753-760.

6. Morente-Sánchez J., Zabala M. // Sports Med. 2013. Vol. 43. pp. 395-411.

7. Temerdashev A.Z., Azaryan A.A., Labutin A.V., Dikunets M.A. et al. // J. Anal. Chem. 2017. Vol. 72. pp. 1184-1192.

\section{References}

1. World Anti-Doping Agency. The World Anti-Doping Code. The 2019 Prohibited List. International Standard. Available at: https://www.wadaama.org/sites/default/files/wada_2019_english_ prohibited_list.pdf (accessed 22 August 2019).

2. Thevis M., Kuuranne T., Geyer H., Drug Test. Anal., 2018, Vol. 10, pp. 9-27. doi: $10.1002 /$ dta. 2336
8. Asatryan B., Vital C., Kellerhals C., Medeiros-Domingo A. et al. // PLOS ONE. 2017. Vol. 12. pp. e0174434.

9. Halabchi F., Seif-Barghi T., Mazaheri R., Asian J. // Sports Med. 2011. Vol. 2. pp. 1-15.

10.Tiscione N.B., Alford I., Yeatman D.T., Shan X. // J. Anal. Tox. 2011. Vol. 35. pp. 501511.

11.Kristoffersen L., Stormyhr L.-E., SmithKielland A. // Forensic Sci. Int. 2006. Vol. 161. pp. 151-157.

12.Shan X., Tiscione N.B., Alford I., Yeatman D.T. // Forensic Sci. Int. 2011. Vol. 211. pp. 4750.

13.Wasfi I.A., Al-Awadhi A.H., Al-Hatali Z.N., Al-Rayami F.J. et al. // J. Chromatogr. B. 2004. Vol. 799. pp. 331-336.

14.WADA Technical Document TD2016EAAS. Endogenous Anabolic Androgenic Steroids Measurement and Reporting. Режим доступа: https://www.wadaama.org/sites/default/files/resources/files/wadatd2016eaas-eaas-measurement-and-reportingen.pdf (дата обращения: 22.08.2019).

3. De Cock K.J.S., Delbeke F.T., Van Eenoo P., Desmet N. et al., J. Pharm. Biomed. Anal., 2001, Vol. 25, pp. 843-852. doi: 10.1016/S0731-7085(01)00396-X

4. de Hon O., Coumans B., British J. Sports Med., 2007, Vol. 41, pp. 800-805. doi:10.1136/bjsm.2007.037226 
5. Furjan Mandic G., Peric M., Krzelj L., Stankovic S. et al., J. Sports Sci. Med., 2013, Vol. 12, pp. 753-760.

6. Morente-Sánchez J., Zabala M., Sports Med., 2013, Vol. 43, pp. 395-411. doi:10.1007/s40279-013-0037-x

7. Temerdashev A.Z., Azaryan A.A., Labutin A.V., Dikunets M.A. et al., J. Anal. Chem., 2017, Vol. 72, pp. 1184-1192. doi:10.1134/s1061934817110090

8. Asatryan B., Vital C., Kellerhals C., Medeiros-Domingo A., Grani C., Trashsel L.D., Schied C.M., Saguner A.M., Eser P., Herzig D., Bolliger S., Michaud K., Willhelm M., PLOS ONE, 2017, Vol. 12, pp. e0174434. doi:10.1371/journal.pone.0174434

9. Halabchi F., Seif-Barghi T., Mazaheri R., Asian J. Sports Med., 2011, Vol. 2, pp. 1-15.

10. Tiscione N.B., Alford I., Yeatman D.T., Shan X., J. Anal. Tox., 2011, Vol. 35, pp. 501511. doi:10.1093/anatox/35.7.501

Темердашев Азамат Зауалевич - доцент кафедры аналитической химии, к.х.н., Кубанский государственный университет, Краснодар

Гашимова Элина Мансуровна - аспирант кафедры аналитической химии, Кубанский государственный университет, Краснодар
11. Kristoffersen L., Stormyhr L.-E., SmithKielland A., Forensic Sci. Int., 2006, Vol. 161, pp. 151-157. doi:10.1016/j.forsciint. 2006.03.034

12. Shan X., Tiscione N.B., Alford I., Yeatman D.T., Forensic Sci. Int., 2011, Vol. 211, pp. 47-50. doi: 10.1016/j.forsciint.2011.04.012.

13. Wasfi I.A., Al-Awadhi A.H., Al-Hatali Z.N., Al-Rayami F.J. et al., J. Chromatogr. B, 2004, Vol. 799, pp. 331-336. doi:10.1016/j.jchromb.2003.11.003

14. WADA Technical Document TD2016EAAS. Endogenous Anabolic Androgenic Steroids Measurement and Reporting. Available at: https://www.wadaama.org/sites/default/files/resources/files/wadatd2016eaas-eaas-measurement-and-reportingen.pdf (accessed 19 August 2019)

Temerdashev Azamat Z. - assistant professor, Ph.D., analytical chemistry department, Kuban State University, Krasnodar, e-mail: TemerdashevAZ@gmail.com

Gashimova Elina M. - Ph.D. student, analytical chemistry department, Kuban State University, Krasnodar, e-mail: elina.gashimova@yandex.ru 